\title{
SwitchPaD: Active Lateral Force Feedback over a Large Area Based on Switching Resonant Modes
}

\author{
Heng $\mathrm{Xu}{ }^{(凶)}$, Michael A. Peshkin, and J. Edward Colgate \\ The Department of Mechanical Engineering, Northwestern University, \\ Evanston, IL 60208-3111, USA \\ hengxu@u.northwestern.edu, \{peshkin, colgate\}@northwestern.edu
}

\begin{abstract}
We present a new device, the SwitchPaD, to generate an active lateral force on a bare fingertip over a large touch area. Like our previous device, the UltraShiver, the SwitchPaD uses synchronization of in-plane ultrasonic oscillation and out-of-plane electroadhesion to generate force. The UltraShiver, however, relied on a single longitudinal resonance to produce oscillations, resulting in an inconsistent force profile. The SwitchPaD switches between the first and the second longitudinal mode based on the finger position, resulting in a much more consistent force profile across the touch surface. Experiments are used to compare the performance of two different modal switching strategies. Results indicate that the SwitchPaD can generate $250 \mathrm{mN}$ peak active lateral force over a large area, and that, with the proper switching strategy, the switch itself is imperceptible.
\end{abstract}

Keywords: Active lateral force feedback · Resonant modes switch · Ultrasonic oscillation · Electroadhesion

\section{Introduction}

Compared with friction modulation methods (ultrasonic friction modulation [1, $2]$, electroadhesion [3,4]), a surface providing active lateral force feedback may render a wider range of haptic effects. Included are effects such as potential well rendering [5] that require propulsive forces, and effects such as button click rendering [6] that require active forces on a stationary finger. In an effort to realize these benefits, a variety of interesting devices have been developed with the goal of providing active lateral force feedback. For instance, Gueorguiev et al. [7] proposed a traveling-wave based device that could provide $100 \mathrm{mN}$ active lateral force with a pressing force of $0.5 \mathrm{~N}$. Since devices employing traveling waves typically operate off resonance, a bulky actuator was required to generate strong lateral forces for haptic rendering. Our group has developed a range of resonant devices that can provide active lateral force, including the ShiverPaD [8] and eShiver [9]. In those devices (and a similar device in [10]), the touch surface 
was oscillated by a voice coil actuator. Even though this approach generated a strong lateral force, the voice coil actuators were bulky, and the resonant frequencies were within the range of human hearing, resulting in noise.

The UltraShiver, for the first time, combined piezoelectric excitation of an ultrasonic resonance with electroadhesion to achieve both high forces and silent operation [11], but provided only a small touch area. This paper proposes a new haptic device, the SwitchPaD, that can achieve active lateral forces $( \pm 250 \mathrm{mN})$ over a large area and in the inaudible region. It oscillates an electroadhesive surface in-plane by selectively exciting the first $(22,390 \mathrm{~Hz})$ or the second $(53,320 \mathrm{~Hz})$ resonant longitudinal mode based on the finger position. Experiments described in Sect. 5 were used to evaluate two different modal switching strategies and to investigate the ability of the SwitchPaD to provide consistent lateral force over a large area.

\section{Background and Motivation}

The UltraShiver consists of two piezoelectric actuators glued symmetrically on opposite sides of a sheet of anodized aluminum [11]. Forces are produced by synchronizing in-plane ultrasonic oscillation and out-of-plane electroadhesion. The former is tuned to the first longitudinal resonance of the UltraShiver. Even though the device shows good ability to control the lateral force, render convincing haptic effects, and localize them $[6,11]$, it cannot provide a consistent magnitude of the lateral force over a large area due to the mode shape. The lateral force decreases to zero as the finger moves toward the nodal line, which is close to the center of the surface.

To remove this limitation, one possible solution is to take advantage of the second longitudinal resonance of the surface, because the anti-node line of the second mode lies at the same location as the node line of the first mode. In general, there are two methods: two-mode superposition and two-mode switching. In modal superposition, the first mode and the second mode are excited at the same time. Unfortunately, the combination of two modes produces a complicated spatiotemporal variation of the surface velocity, making it difficult to synchronize properly with electroadhesion. Additionally, due to the limitation of the power source and the PZT material, two modes cannot be excited fully at the same moment. They have to share the total power and the ability of the PZT material.

For these reasons, we chose to explore the modal switching strategy. In this strategy, when the finger slides from one end of the surface to the other, crossing the boundary where the lateral force generated by the second mode starts to be greater than that by the first mode, the resonance is switched from the first mode to the second. We call this approach the "SwitchPaD". The challenge here is how to design the SwitchPaD with optimal Q factors for each resonance so that it can generate high lateral force (greater than $125 \mathrm{mN}$ peak), yet switch modes without perceptual artifact. 


\section{Method and Experiment}

\subsection{SwitchPaD Design}

Structure. The structure of the SwitchPaD (as shown in Fig. 1 and Fig. 2) is similar to the UltraShiver (shown in [11]), and consists of two piezoelectric disc actuators and a sheet of anodized aluminum. The dimensions of the anodized aluminum are $104 \times 22 \times 1 \mathrm{~mm}$. The two soft piezos (SMD22T25R211WL, Steminc and Martins Inc, Miami, FL, USA) are $22 \mathrm{~mm}$ diameter $\times 0.25 \mathrm{~mm}$ thick. They are excited in-phase with one another to provide strong coupling to the longitudinal rather than flexural modes, and they are located $26 \mathrm{~mm}(=104 \mathrm{~mm} / 4)$ from the edge of the aluminum plate as a "sweet spot" for exciting both modes. This location ensures that the surface can generate enough lateral velocity in both modes and also attenuate quickly during the switch. This "sweet spot" was optimized via a series of FEA simulations and experiments which are beyond the scope of this paper.

Lateral Force Generation Principle. A detailed model of force generation with the UltraShiver is given in [11]. The SwitchPaD produces lateral force in the same way: as a result of friction being greater when electroadhesion is turned high than when it is turned low, and this effect being synchronized with in-plane oscillation. The direction and magnitude of the lateral force can be adjusted by varying the phase between the oscillation and the electroadhesion. The SwitchPaD is different, however, insofar as the resonant mode is switched depending on finger position. This ensures a high oscillation velocity wherever the finger may be on the touch surface.

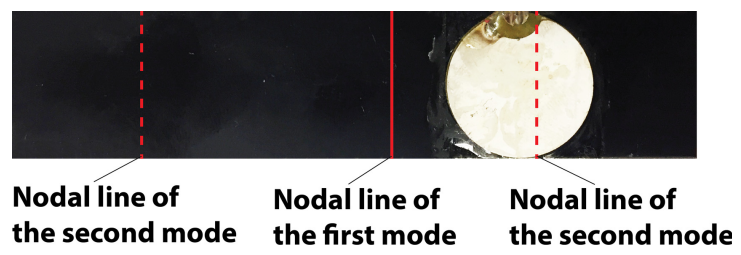

Fig. 1. Top view of the SwitchPaD.

\subsection{Experiment Setup}

Figure 2 shows the experiment setup, in which the SwitchPaD was mounted to mechanical ground (acrylic block) with four brass flexures, and the electrically grounded index finger of the dominant hand was constrained to move only up and down.

Two different sensors were used in this experiment setup, including a six-axis force sensor (ATI Nano 17 Force/Torque sensor) and a Laser Doppler Vibrometer 
(LDV, IVS-500, Polytec, Inc). The force sensor was used to measure the lateral force on the surface and the LDV was used to measure the lateral velocity at the end of the surface (in Sect. 5.1) or at the side of the finger (in Sect. 5.2). In addition, a CCD sensor was used to track the finger position. The setup in Fig. 2 was placed on a linear rail controlled by a DC motor, which was used to move the SwitchPaD at a constant velocity (more details in Sect. 4.1 and 5.2).

The piezoelectric actuator voltage and the electroadhesive current were controlled with a custom voltage amplifier and a custom transconductance amplifier, respectively (more details were reported in [11,12]). All signals were recorded using a NI USB-6361 Multifunctional I/O Device with a $200 \mathrm{kHz}$ sampling frequency.

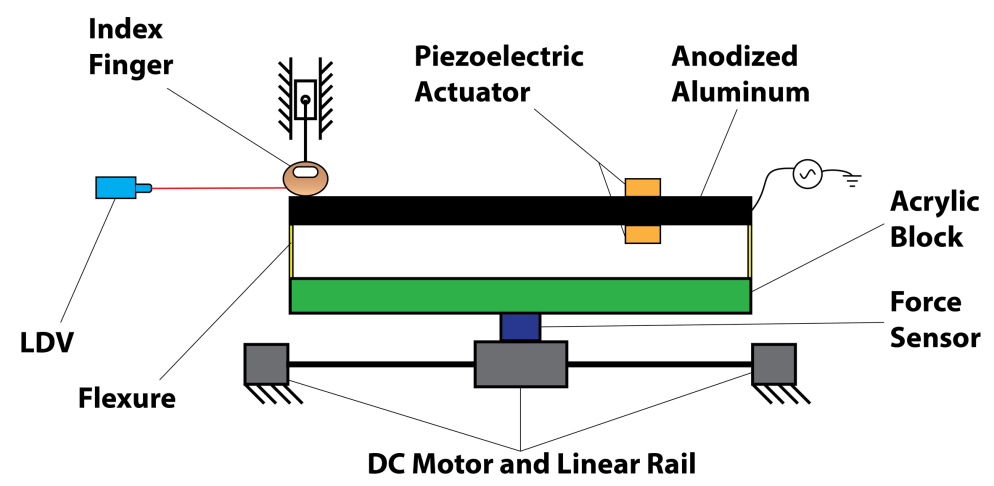

Fig. 2. Experiment platform.

\section{Force Profile Measurement for Each Mode}

\subsection{Experiment Protocol}

As a first experiment, the lateral force profile (force as a function of finger position) generated by each resonant mode was measured. The frequencies of the first and second modes were $22,390 \mathrm{~Hz}$ and $53,320 \mathrm{~Hz}$, respectively. The input voltage of the piezoelectric actuator and electroadhesion were $30 \mathrm{~V}$ peak and $100 \mathrm{~V}$ peak with an offset of $200 \mathrm{~V}$, respectively. The phase between the piezoelectric voltage and the electroadhesive voltage was set to generate peak lateral force (in Fig. 2).

During the experiments, the electrically grounded finger lightly touched the surface and kept a constant pressing force $(0.3 \mathrm{~N})$ as effectively as possible while the SwitchPaD was moved at $50 \mathrm{~mm} / \mathrm{s}$ by the DC motor and the linear rail. The finger moved from the left end of the surface to the left side of the piezoelectric actuator. Each resonant mode was excited in one trial individually, and each trial was repeated ten times. 


\subsection{Results and Discussions}

As shown in Fig. 4, both the first mode and the second mode can achieve around $300 \mathrm{mN}$ active lateral force, but at different positions. For the first mode (blue curve in Fig. 4), the lateral force keeps a constant value (around $300 \mathrm{mN}$ ) from $0 \mathrm{~mm}$ to $25 \mathrm{~mm}$ and decreases to zero at $60 \mathrm{~mm}$, which is consistent with the model prediction in [11]. For the second mode (red curve in Fig. 4), the lateral force is approximately constant from $25 \mathrm{~mm}$ to $65 \mathrm{~mm}$. Thus, these results suggest that combining the first mode and the second mode is a promising method to provide a constant lateral force from $0 \mathrm{~mm}$ to $65 \mathrm{~mm}$.

\section{Resonant Mode Switch}

Based on the results in Fig. 4, a switch located anywhere from $25 \mathrm{~mm}$ to $41 \mathrm{~mm}$ could be used to achieve an approximately constant lateral force (around 250 $\mathrm{mN}$ ). In this section, the switch point was placed at $41 \mathrm{~mm}$, and two different modal switching strategies were compared.

\subsection{Mode Switch Strategy}

When the finger slides from the left side to the right side and crosses the switch line $(41 \mathrm{~mm})$, the resonant mode is switched from the first mode to the second mode, vice versa. We explored two different switch strategies: instant and gradual.

For the instant switch, input voltage for the first mode is turned off at the same time input voltage for the second mode is turned on. Thus, there is only one resonant mode being driven at any instant, although there are ring-up $(3.9 \mathrm{~ms})$ and ring-down $(6.9 \mathrm{~ms})$ times for both modes due to their high $\mathrm{Q}$ values (first mode: 482 , second mode: 651 ). When the LDV was used to measure the lateral velocity at the left end of the surface (in Fig. 2), a spike of the lateral velocity was found at the switch point (shown as the blue solid and dashed curves in Fig. 3(a)). The spike is due to the ring-up and ring-down times and is strongly perceived by the subject. This perceptual artifact makes the instant switch unacceptable for applications that require continuous force feedback on the surface.

To avoid the spike at the switch point, the idea of a gradual switch was investigated. In this strategy, the input voltage to the piezoelectric actuator at the first mode decreases gradually, and at the same time, the input voltage at the second mode increases gradually. This process takes around $100 \mathrm{~ms}$, a time frame that was roughly optimized via simulation (see Fig. 3(b)). During this transition period, both resonant modes were excited, and electroadhesion was also operated at both frequencies with amplitude ramps that tracked those of the piezoelectric input voltages. The experiment results (red solid and dashed curves in Fig. 3(a)) show good agreement with the simulation results. More importantly, lateral force was preserved and the gradual switch could not be perceived. 


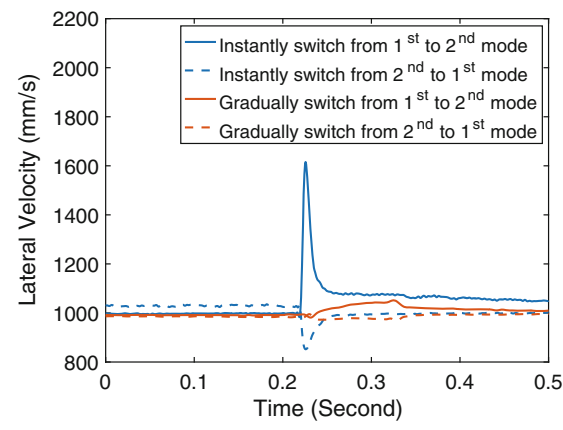

(a) Lateral velocity measurement of the instant switch and the gradual switch

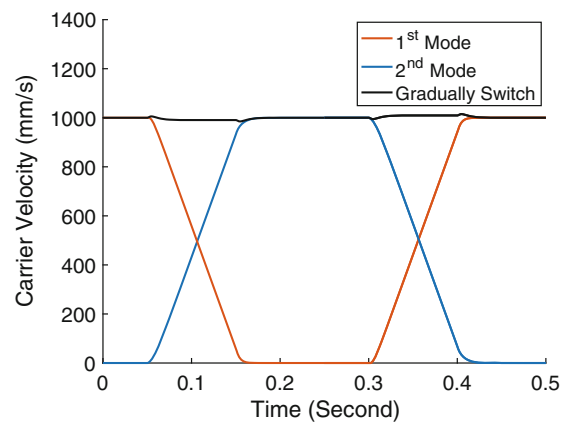

(b) Simulation of the gradual switch

Fig. 3. Lateral Velocity simulation and measurement during switches. All the lateral velocity simulations and measurements are at the left end of the surface. (Color figure online)

\subsection{Experiment Protocol}

This experiment is similar to that in Sect.4.1, however, the LDV was used to measure the lateral velocity at the left side of the finger while the force sensor measured the lateral force on the surface. Experiments were performed with the two different switch strategies and repeated ten times.

\subsection{Results and Discussions}

The results are shown as force sensor measurements (in Fig. 4) and LDV measurements (in Fig. 5). Figure 4 shows that both the instant switch and the gradual switch can generate constant lateral force (around $250 \mathrm{mN}$ ) from $0 \mathrm{~mm}$ to $65 \mathrm{~mm}$. The bandwidth of the force sensor, however, is below $50 \mathrm{~Hz}$, so that it unable to detect rapid transients. LDV measurements instead were used to investigate performance up to $10 \mathrm{kHz}$.

Since the whole experiment setup was moved by a DC motor and a linear rail, which was operated via open-loop control of the velocity (more details in Sect.4.1), the five trials in Fig. 5 do not align temporally. Figure 5(a) shows that, in every trial, there is a spike of the finger motion. This spike occurs when the finger crosses the mode switch line $(41 \mathrm{~mm})$. The peak velocity of this spike is around $35 \mathrm{~mm} / \mathrm{s}$ with $10 \mathrm{~ms}$ width, which is above the human detection threshold of vibration $[13,14]$. In contrast to the instant switch, velocity spikes are essentially absent during the gradual switch. The lateral velocity varies within $\pm 5 \mathrm{~mm} / \mathrm{s}$ with $40 \mathrm{~ms}$ width, which cannot be perceived by subjects.

Thus, the instant switch strategy can only be used for specific haptic applications, such as button click rendering, in which a spatially discontinuous lateral force is acceptable. Since the gradual switch strategy can provide consistent lateral force over a large area, it can be used for more general haptic applications, such as shape rendering. 


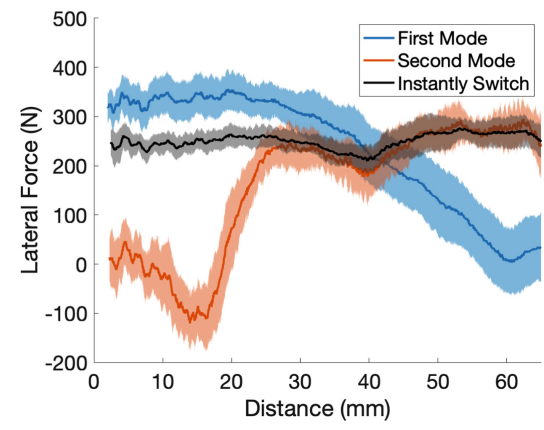

(a) Instantly switch

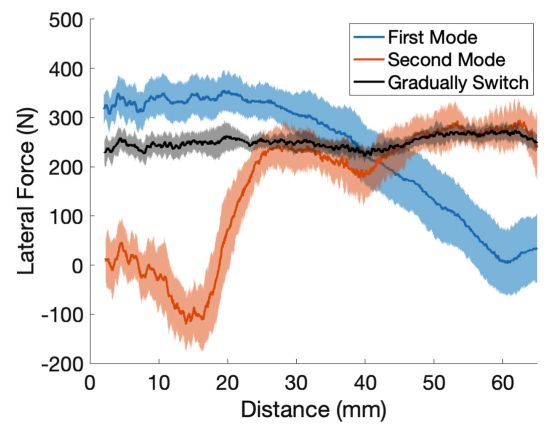

(b) Gradually switch

Fig. 4. Lateral force measurement on the surface. The $\mathrm{x}$-axis shows the distances from the left end of the surface. The solid curves and the shadows are the averages and standard deviations over ten trials at the corresponding experiment condition (the first mode, the second mode, the instant switch, and the gradual switch).

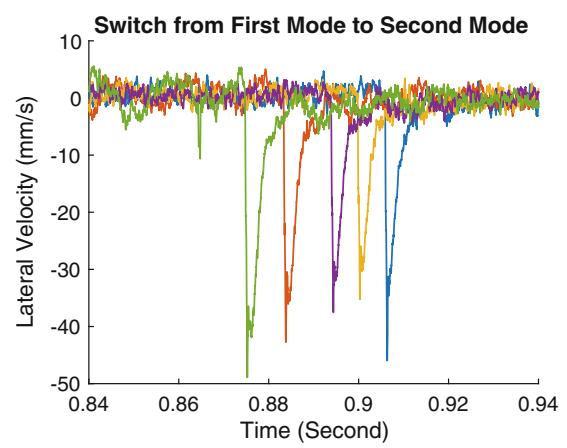

(a) Instant switch

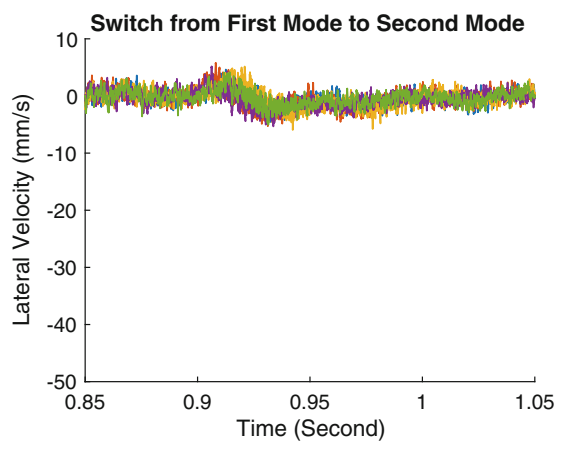

(b) Gradual switch

Fig. 5. Lateral velocity measurement at the left side of fingertip during instant switches and gradual switches. Each colored curve represents one trial. (Color figure online)

\section{Conclusion}

The SwitchPaD presented in this paper employs the first $(22,390 \mathrm{~Hz})$ and second $(53,320 \mathrm{~Hz})$ resonant longitudinal modes to achieve a strong active lateral force. By gradually switching between the first and second modes when the finger crosses a threshold position, the SwitchPaD can keep the lateral force consistent (250 mN peak) over a large area. This significantly improves the ability to render more general haptic effects compared to our previous device, the UltraShiver [11].

Acknowledgment. This material is based upon work supported by the National Science Foundation grants number IIS-1518602. 


\section{References}

1. Watanabe, T., Fukui, S.: A method for controlling tactile sensation of surface roughness using ultrasonic vibration. In: Proceedings of 1995 IEEE International Conference on Robotics and Automation 1995, vol. 1, pp. 1134-1139. IEEE (1995)

2. Winfield, L., Glassmire, J., Colgate, J.E., Peshkin, M.: T-PaD: tactile pattern display through variable friction reduction. In: Second Joint EuroHaptics Conference and Symposium on Haptic Interfaces for Virtual Environment and Teleoperator Systems (WHC 2007), pp. 421-426. IEEE (2007)

3. Linjama, J., Mäkinen, V.: E-sense screen: novel haptic display with capacitive electrosensory interface. In: 4th Workshop for Haptic and Audio Interaction Design HAID 2009 (2009)

4. Shultz, C.D., Peshkin, M.A., Colgate, J.E.: Surface haptics via electroadhesion: expanding electrovibration with Johnsen and Rahbek. In: 2015 IEEE World Haptics Conference (WHC), pp. 57-62. IEEE (2015)

5. Osgouei, R.H., Kim, J.R., Choi, S.: Improving 3D shape recognition with electrostatic friction display. IEEE Trans. Haptics 10(4), 533-544 (2017)

6. Xu, H., Klatzky, R.L., Peshkin, M.A., Colgate, E.: Localizable button click rendering via active lateral force feedback. IEEE Trans. Haptics (2020)

7. Ghenna, S., Vezzoli, E., Giraud-Audine, C., Giraud, F., Amberg, M., LemaireSemail, B.: Enhancing variable friction tactile display using an ultrasonic travelling wave. IEEE Trans. Haptics 10(2), 296-301 (2016)

8. Chubb, E.C., Colgate, J.E., Peshkin, M.A.: ShiverPaD: a glass haptic surface that produces shear force on a bare finger. IEEE Trans. Haptics 3(3), 189-198 (2010)

9. Mullenbach, J., Peshkin, M., Colgate, J.E.: eShiver: lateral force feedback on fingertips through oscillatory motion of an electroadhesive surface. IEEE Trans. Haptics 10(3), 358-370 (2016)

10. Alma, U.A., Ilkhani, G., Samur, E.: On generation of active feedback with electrostatic attraction. In: Bello, F., Kajimoto, H., Visell, Y. (eds.) EuroHaptics 2016. LNCS, vol. 9775, pp. 449-458. Springer, Cham (2016). https://doi.org/10.1007/ 978-3-319-42324-1_44

11. Xu, H., Peshkin, M.A., Colgate, J.E.: UltraShiver: lateral force feedback on a bare fingertip via ultrasonic oscillation and electroadhesion. IEEE Trans. Haptics 12(4), 497-507 (2019)

12. Shultz, C., Peshkin, M., Colgate, J.E.: The application of tactile, audible, and ultrasonic forces to human fingertips using broadband electroadhesion. IEEE Trans. Haptics 11(2), 279-290 (2018)

13. Mountcastle, V.B.: The neural replication of sensory events in the somatic afferent system. In: Eccles, J.C. (ed.) Brain and Conscious Experience, vol. pp. 85-115. Springer, Heidelberg (1965). https://doi.org/10.1007/978-3-642-49168-9_4

14. Kandel, E.R., Schwartz, J.H., Jessell, T.M., Siegelbaum, S., Hudspeth, A.J.: Principles of Neural Science, vol. 4. McGraw-hill, New York (2000) 
Open Access This chapter is licensed under the terms of the Creative Commons Attribution 4.0 International License (http://creativecommons.org/licenses/by/4.0/), which permits use, sharing, adaptation, distribution and reproduction in any medium or format, as long as you give appropriate credit to the original author(s) and the source, provide a link to the Creative Commons license and indicate if changes were made.

The images or other third party material in this chapter are included in the chapter's Creative Commons license, unless indicated otherwise in a credit line to the material. If material is not included in the chapter's Creative Commons license and your intended use is not permitted by statutory regulation or exceeds the permitted use, you will need to obtain permission directly from the copyright holder. 